VOLUMe 28 (2020) p. 229

DOI: $10.24330 /$ ieja. 768272

\title{
AN ADDENDUM TO THE PAPER: MODULES WITH FINITELY MANY SUBMODULES
}

\author{
Gabriel Picavet and Martine Picavet-L'Hermitte \\ Received: 19 May 2020; Accepted: 28 May 2020 \\ Communicated by Abdullah Harmancı
}

In our paper entitled: Modules with finitely many submodules, [2], the statement of [2, Theorem 2.13] does not seem clear enough for some readers. So we supply some more explanations for this statement, which gives the structure of FMS faithful modules (FMS stands for finitely many submodules). The proof relies on a result of Anderson and Chun, [1, Corollary 2.4], that characterizes rings with finitely many ideals. These authors do not consider the zero ring, which is trivially a ring with finitely many ideals. Now observe that any $\operatorname{ring} R$ is isomorphic to the ring $R \times 0$, so that any ring is (isomorphic to) a direct product of two rings. This simple fact already answers some questions. Using the result of Anderson and Chun, we can write $R \simeq R^{\prime} \times R^{\prime \prime}$ for some rings $R^{\prime}$ and $R^{\prime \prime}$, such that $\left|R^{\prime}\right|<\infty$ and $\left|R^{\prime \prime} / P\right|=\infty$ for each $P \in \operatorname{Spec}\left(R^{\prime \prime}\right)$. But we have neglected to consider the routine following cases. If $R^{\prime}$ does not appear (that is $R=R^{\prime \prime}$ ), it is enough to take $R^{\prime}=0$. If $R^{\prime \prime}$ does not appear (that is $R=R^{\prime}$ ), it is enough to take $R^{\prime \prime}=0$, because the spectrum of a zero ring is empty and the $R^{\prime \prime}$-module $M^{\prime \prime}$ being zero appears as a projective module of rank one. Then the reader may verify that the proof is valid.

\section{References}

[1] D. D. Anderson and S. Chun, Commutative rings with finitely generated monoids of fractional ideals, J. Algebra, 320 (2008), 3006-3021.

[2] G. Picavet and M. Picavet-L'Hermitte, Modules with finitely many submodules, Int. Electron. J. Algebra, 19 (2016), 119-131.

\author{
Gabriel Picavet (Corresponding Author) and Martine Picavet-L'Hermitte \\ Mathématiques, 8 Rue du Forez \\ 63670 Le Cendre, France \\ emails: picavet.mathu@orange.fr (G. Picavet) \\ picavet.mg@orange.fr (M. Picavet-L'Hermitte)
}

